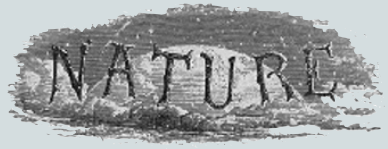

50 YEARS AGO

"A Suggested Revision of Nomenclature - Angiotensin" The vasoactive peptide resulting from the action of rennin on an alpha-globulin was discovered by two groups of investigators, with the result that it received two trivial names, angiotonin and hypertensin. Synthesis of the octapeptide has now confirmed the identity of this peptide and justifies dropping the double nomenclature. We propose the simplified name, angiotensin, and its derivatives angiotensinase and angiotensinogen. Angiotensin is a hybrid word but does, we think, have the advantage of being easy to pronounce even with a variety of accents, is euphonious, and is understandable despite the most recalcitrant microphone. Eduardo Braun-Menendez \& Irvine H. Page From Nature 12 April 1958.

\section{YEARS AGO}

Paris, Academy of Sciences, March 30 ... The determination of time, both on land and at sea, with the aid of wireless telegraphy: Bouquet de la Grye. With the present installation at the Eiffel Tower, wireless signals can be sent a distance of 2000 kilometres, and it has been estimated that by increasing the electric energy this distance could be doubled. It is suggested that a special signal should be sent exactly at midnight ... Such a time signal would be of the greatest service to navigators within its radius. On the proposal of the president, the examination of the proposition ... was referred to a committee composed of the members of the sections of astronomy, geography, navigation, and physics, together with MM. Darboux, Poincaré, and Cailletet.

A phenomenon attributable to positive electrons in the spark spectrum of yttrium: Jean Becquerel. The experimental study of the Zeeman effect in the spark spectrum of yttrium gives results which may be most simply explained by assuming the presence of positive electrons. From Nature 9 April 1908.

\title{
a Early hominin
}

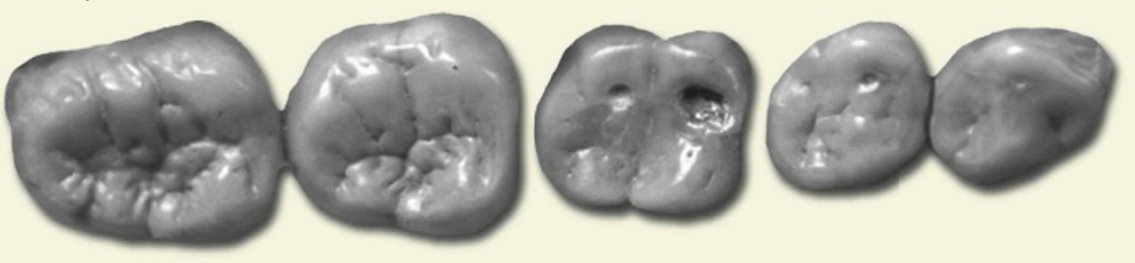

b Chimpanzee

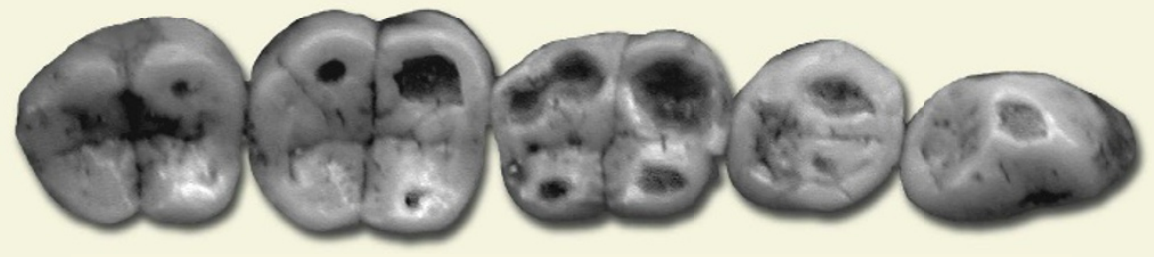

Figure 1 | Shear quality. Comparisons of moderately worn premolar and molar crown surfaces of (a) an early hominin, Australopithecus afarensis (fossil AL266, dating to about 3.3 million years ago), and (b) a chimpanzee. Wear of thin enamel, as seen in the chimpanzee, quickly leads to sharp edges where the hard outer surface gives way to softer, underlying dentine. The hominin molar is flatter, with thicker enamel, and wear generally does not produce sharp edges. Chimpanzee teeth may be better suited to efficient shearing or grinding of softer, tougher foods than to crushing of hard, brittle items.

premolars and molars, given food with specific fracture properties. The principal job of a tooth is to fracture foods, and teeth can be stressed by considerable, repeated loading forces as they go about the business of chewing. Teeth must break foods without themselves being broken; they do not 'heal', and the teeth of most adult mammals cannot be replaced.

Starting from these premises, Lucas et al. offer several predictions. The first relates to the fact that tooth crowns are bilayered, with hard, brittle enamel overlying more compliant dentine. A thin enamel coat would be more prone to flex and cause tensile stresses within the tooth, leading to cracks that would start at the undersurface of the crown and spread outwards. Thicker enamel would provide one way to resist tooth fracture caused by the high stresses associated with feeding on hard objects. Changing the structure of the enamel would also help. Enamel forms as rods or prisms that align together like bunches of dry spaghetti strands. The cap is better able to resist forces oriented parallel to the long axis of these prisms than perpendicular to them. Furthermore, cracks spreading between adjacent prisms may be stopped if the rods change direction periodically (a phenomenon known as decussation).

Lucas et al. propose decussation as a mechanism for strengthening teeth that have enamel of uneven thickness. As an example of another strengthening mechanism, soft foods would be expected to 'smother' a tooth surface, redistributing tensile forces to the margins. This might explain why some mammals possess a cingulum, a collar of enamel around the periphery of the crown.

The authors also consider fracture resistance from the food's perspective. It does a plant little good to have its seeds destroyed, so seed coats should also evince adaptations to resist breakage. Such adaptations can be remarkably similar to those seen in teeth, given that both tooth crowns and seed shells are often bilayered. This leads to predictions for shell thickness and internal-fibre decussation that are analogous to those seen in teeth. Tooth-food interactions can thus be viewed as a 'death match' as nature selects for strength in both dental enamel and seed shells.

The application of principles from fracture and deformation mechanics to address the relationships between tooth and food structures opens avenues for productive research. There have been debates about the best way to measure enamel thickness, given its uneven distribution across a crown ${ }^{4,5}$. A materials-science approach, combined with new imaging technologies such as micro-computed tomography, could help in the development of a standard, functionally relevant protocol. It could also help in formulating computer simulations known as finite-element models that attempt to illuminate the relationships between tooth form and chewing stress ${ }^{6}$, as both methods aim to elucidate how teeth and foods react when the jaws close on an item of food.

Teeth are highly complicated structures, and much remains to be done if we are to understand the adaptive significance of mammalian dental form. In terms of enamel structure, functionally relevant aspects of complexity occur at many different levels: crystallites form prisms, prisms are organized into enamel types, and enamel types are combined into schmelzmusters, or layers ${ }^{7}$. These must ultimately be built into the models 